\title{
Identifying the Dominant Contribution of Human Cytochrome P450 2J2 to the Metabolism of Rivaroxaban, a New Oral Anticoagulant
}

\author{
Tingting Zhao ${ }^{1}$, Yanwei Chen ${ }^{2}$, Dalong Wang ${ }^{1}$, Liyan Wang ${ }^{2}$, Peipei Dong ${ }^{1}$, Shan Zhao ${ }^{3}$, \\ Changyuan Wang ${ }^{1}$, Qiang Meng ${ }^{1}$, Huijun Sun ${ }^{1}$, Kexin Liu ${ }^{4}$, and Jingjing Wu ${ }^{1}$ \\ ${ }^{1}$ Dalian Medical University \\ ${ }^{2}$ First Affiliated Hospital of Dalian Medical University \\ ${ }^{3}$ Dalian Institute of Chemical Physics \\ ${ }^{4}$ Affiliation not available
}

October 1, 2020

\begin{abstract}
Aim Rivaroxaban, an oral anticoagulant, undergoes the metabolism mediated by human cytochrome P450 (CYP). The present study is to quantitatively analyze and compare the contributions of multiple CYPs in the metabolism of rivaroxaban to provide new information for medication safety. Methods The metabolic stability of rivaroxaban in the presence of human liver microsomes (HLMs) and recombinant CYPs was systematically evaluated to estimate the participation of various CYP isoforms. Furthermore, the catalytic efficiency of CYP isoforms was compared via metabolic kinetic studies of rivaroxaban with recombinant CYP isoenzymes, as well as via CYP-specific inhibitory studies. Additionally, docking simulations were used to illustrate molecular interactions. Results Multiple CYP isoforms were involved in the hydroxylation of rivaroxaban, with decreasing catalytic rates as follows: CYP2J2 $>3 \mathrm{~A} 4>2 \mathrm{D} 6>4 \mathrm{~F} 3>1 \mathrm{~A} 1>3 \mathrm{~A} 5>3 \mathrm{~A} 7>2 \mathrm{~A} 6>2 \mathrm{E} 1>2 \mathrm{C} 9>2 \mathrm{C} 19$. Among the CYPs, 2J2, 3A4, 2D6 and 4F3 were the four major isoforms responsible for rivaroxaban metabolism. Notably, the intrinsic clearance of rivaroxaban catalyzed by CYP2J2 was nearly 39-, 64- and 100-fold that catalyzed by CYP3A4, 2D6 and 4F3, respectively. In addition, rivaroxaban hydroxylation was inhibited by $41.1 \%$ in the presence of the CYP2J2-specific inhibitor danazol, which was comparable to the inhibition rate of $43.3 \%$ by the CYP3A-specific inhibitor ketoconazole in mixed HLMs. Furthermore, molecular simulations showed that rivaroxaban principally bound to CYP2J2 by $\pi$-alkyl bonds, carbon-hydrogen bonds and alkyl interactions. Conclusion CYP2J2 dominated the hydroxylation of rivaroxaban, which may provide new insight into clinical drug interactions involving rivaroxaban.
\end{abstract}

\section{Methods}

The metabolic stability of rivaroxaban in the presence of human liver microsomes (HLMs) and recombinant CYPs was systematically evaluated to estimate the participation of various CYP isoforms. Furthermore, the catalytic efficiency of CYP isoforms was compared via metabolic kinetic studies of rivaroxaban with recombinant CYP isoenzymes, as well as via CYP-specific inhibitory studies. Additionally, docking simulations were used to illustrate molecular interactions.

\section{Results}

Multiple CYP isoforms were involved in the hydroxylation of rivaroxaban, with decreasing catalytic rates as follows: CYP2J2 $>3 \mathrm{~A} 4>2 \mathrm{D} 6>4 \mathrm{~F} 3>1 \mathrm{~A} 1>3 \mathrm{~A} 5>3 \mathrm{~A} 7>2 \mathrm{~A} 6>2 \mathrm{E} 1>2 \mathrm{C} 9>2 \mathrm{C} 19$. Among the CYPs, 2J2, 3A4, 2D6 and 4F3 were the four major isoforms responsible for rivaroxaban metabolism. Notably, the intrinsic clearance of rivaroxaban catalyzed by CYP2J2 was nearly 39-, 64- and 100-fold that 
catalyzed by CYP3A4, 2D6 and 4F3, respectively. In addition, rivaroxaban hydroxylation was inhibited by $41.1 \%$ in the presence of the CYP2J2-specific inhibitor danazol, which was comparable to the inhibition rate of $43.3 \%$ by the CYP3A-specific inhibitor ketoconazole in mixed HLMs. Furthermore, molecular simulations showed that rivaroxaban principally bound to CYP2J2 by $\pi$-alkyl bonds, carbon-hydrogen bonds and alkyl interactions.

\section{Conclusion}

CYP2J2 dominated the hydroxylation of rivaroxaban, which may provide new insight into clinical drug interactions involving rivaroxaban.

\section{Introduction}

Rivaroxaban, an outstanding representative of a non-vitamin $\mathrm{K}$ oral anticoagulant, directly inhibits Factor $\mathrm{Xa}$ to block the production and reduce the activation of thrombin [1]. Compared with vitamin $\mathrm{K}$ anticoagulants, rivaroxaban exerts a more specific and powerful anticoagulant effect, and has been approved mainly for treatment and prevention of deep venous thrombosis, pulmonary embolism and systemic embolism from nonvalvular atrial fibrillation [2]. In the evaluation of safety and pharmacokinetic stability, rivaroxaban surpasses established anti-coagulant agents; however, bleeding risk still exits [3]. A systematic review and meta-analysis of the efficiency and safety of direct oral anticoagulants approved for treating or preventing cardiovascular thromboembolism complications showed that rivaroxaban did not outperform warfarin in terms of gastrointestinal bleeding risk [4]. Indeed, several bleeding events have been reported when rivaroxaban was applied to prevent stroke and systemic embolism for atrial fibrillation patients, especially when used in combination with other heart rate control drugs [5-7].

Previous studies have investigated the metabolism and elimination of rivaroxaban, with cytochrome P450 (CYP) enzymes, mainly CYP2J2 and CYP3A4, and a few liver hydrolytic enzymes playing an important role in the deactivation of rivaroxaban [89]. The major metabolites and metabolic pathways were identified by in vitro liver microsome incubation studies, and morpholinone 2- hydroxylation (M1) was identified as the structure of the major rivaroxaban metabolite by $\mathrm{H}^{1}$ NMR analysis (Figure 1) [8]. As previously reported, the proportion of rivaroxaban metabolized by CYP enzymes represents approximately two-thirds of a given dose, and the remaining one-third is eliminated by secretion mediated by P-glycoprotein (P-gp) and breast cancer resistance protein (BCRP) [8 10 11]. Pharmacokinetic interactions between rivaroxaban and drugs for regulating CYP3A4 and P-gp have been extensively evaluated, with outcomes indicating that caution is warranted when it is used concomitantly with strong CYP3A4 and P-gp inhibitors [12-14]. Notably, Mueck et al. found that rivaroxaban co-administrated with strong or moderate CYP3A4 inhibitors-such as clarithromycin and fluconazole-did not cause clinically relevant interactions for rivaroxaban [12 14 15]. In addition, bleeding events do exist for combining with other agents in clinical practice, which are not limited to CYP3A4 and P-gp inhibitors [5-7 16 17]. Taken together, we hypothesize that CYP3A4 is not the predominant isoform involved in the metabolism of rivaroxaban, and that other CYP isoforms likely participate to a larger extent in rivaroxaban morpholine 2-hydroxylation [12 18].

In the present study, we systematically evaluated the participation and contribution of a series of CYP isoforms in the metabolism of rivaroxaban by product formation analysis in human liver microsomes (HLMs) and recombinant human CYPs, as well as CYP-specific inhibition studies.

\section{Methods}

\section{Chemicals and instruments}

Rivaroxaban (>98\%) was obtained from Shanghai Yuanye Bio-Technology Co., Ltd. Danazol was purchased from Med Chem Express (New Jersey, USA). All other CYPs inhibitors were purchased from SigmaAldrich (Missouri, USA). PBS was prepared from dipotassium bisulfate and potassium dihydrogen sulfate in the appropriate proportions and stored at $4{ }^{\circ} \mathrm{C}$ until use. Analytical reagent grade and HPLC grade solvents were from Tedia, Inc. (Ohio, USA). Pooled human liver microsomes were purchased from BioreclamationIVT (Maryland, USA). cDNA-expressed recombinant human CYP3A4 and CYP3A5 were obtai- 
ned from Cypex Ltd (Dundee, UK). cDNA-expressed CYP1A1, CYP1A2, CYP1B1, CYP2A6, CYP2B6, CYP2C8, CYP2C9, CYP2D6, CYP2E1, CYP2J2, CYP3A7, CYP4F2, CYP4F3 and CYP46A1 derived from baculovirus-infected insect cells were obtained from BD Gentest Corp. (Massachusetts, USA). cDNAexpressed CYP2C19 fromEscherichia coli was purchased from New England Biolabs Ltd. (Beijing, China). All microsomal samples and recombinant human $\mathrm{P} 450$ isoforms were stored at $-80{ }^{\circ} \mathrm{C}$ until use.

High-performance liquid chromatography (HPLC) was performed using an Agilent MSD/MS system controller, two 1260 series pumps, a 1200 series autosampler and a 1200 series variable wavelength detector. Other instrumentation included a constant temperature vibrating mixer (Hangzhou ALLSHENG Instrument Co. Ltd, China), scroll machine, centrifuge and elite ODS-BP analysis column $(4.6 \times 200 \mathrm{~mm}, 5 \mu \mathrm{m})$. An API 3200 triple-quadrupole mass spectrometer (Applied Biosystems, Ontario, Canada) was used for LC-MS analysis. Ionization was conducted using an electron spray interface in the positive ion mode for detecting metabolites.

\section{HPLC analysis methodology}

The mobile phase consisted of $60 \%$ methanol (A pump) and $40 \%$ pure water with $0.2 \%$ formic acid (B pump) with isocratic elution. Detection conditions were as follows: column temperature, $30{ }^{\circ} \mathrm{C}$; autosampler injection volume, $20 \mu \mathrm{L}$; flow rate, $0.5 \mathrm{~mL} \cdot \mathrm{min}^{-1}$; and, detector wavelength, $240 \mathrm{~nm}$. The major rivaroxaban metabolite (M1), was quantified from the rivaroxaban standards, for which the correlation coefficient was > 0.999. The quantitative method showed good sensitivity.

\section{Incubation conditions}

The final incubation conditions with HLM and recombinant CYPs were optimized, which were within the linear range for the concentrations of major metabolite that formed. The HLM incubation system, with a total volume of $200 \mu \mathrm{L}$, contained potassium phosphate buffer $(10 \mathrm{mM}, \mathrm{pH} 7.4)$, NADPH $(1 \mathrm{mM})$, and liver microsomes $\left(0.3 \mathrm{mg}\right.$ protein $\left.\cdot \mathrm{mL}^{-1}\right)$. The total volume of the incubation system with recombinant CYPs was set at $100 \mu \mathrm{L}$. All rivaroxaban samples in this study were obtained by a series of dilutions from a stock solution (100 mM in DMSO). The final organic solvent concentration was no more than $0.5 \%(\mathrm{v} / \mathrm{v})$. After 5 minutes of incubation at $37{ }^{\circ} \mathrm{C}$, the reaction was initiated by the addition of NADPH and the resulting mixture incubated at $37^{\circ} \mathrm{C}$ on a vibrating mixer for 60 minutes. The reaction was terminated by the addition of cold methanol in a volume equivalent to that of the reaction system. The reaction mixture was then centrifuged at $2000 \mathrm{x}$ g for 15 minutes. Aliquots of the supernatants were stored at $-20{ }^{\circ} \mathrm{C}$ until analysis by HPLC. The reactions that were incubated without NADPH, substrate or enzymes were designed to confirm the formation of the metabolite was dependent on enzymes and NADPH.

\section{Kinetic analysis}

Rivaroxaban $(1-100 \mu \mathrm{M})$ was incubated with pooled HLM $\left(0.3 \mathrm{mg}\right.$ protein $\left.\cdot \mathrm{mL}^{-1}\right)$ for $60 \mathrm{~min}$ to evaluate the kinetic parameters of the HLM. Rivaroxaban (1-200 $\mu \mathrm{M}$ for CYP2J2, 10-200 $\mu \mathrm{M}$ for CYP 2D6 and 20-200 $\mu \mathrm{M}$ for CYP4F3) was incubated with recombinant CYPs $\left(0.4 \mathrm{mg}\right.$ protein $\left.\cdot \mathrm{mL}^{-1}\right)$ for $60 \mathrm{~min}$ to determine the kinetic parameters of the recombinant CYPs. The incubation conditions were as described above. The apparent $K \mathrm{~m}$ and $V$ max values were calculated from nonlinear regression analysis of experimental data according to the Michaelis-Menten equation with GraphPad Prism, version 7. All incubations were carried out in three independent experiments performed in duplicate.

\section{Assay with recombinant $P 450$ isoforms}

Seventeen cDNA-expressed human P450 isoforms, including CYP1A1, CYP1A2, CYP1B1, CYP2A6, CYP2B6, CYP2C19, CYP2C8, CYP2C9, CYP2D6, CYP2E1, CYP2J2, CYP3A4, CYP3A5, CYP3A7, CYP4F2, CYP4F3 and CYP46A1 were used. Rivaroxaban $(120 \mu \mathrm{M})$ was incubated with recombinant CYPs at $37{ }^{\circ} \mathrm{C}$ for 60 minutes to estimate the participation of the CYP isoforms. Results were from at least three experiments carried out in duplicate. The quantitative determination of metabolite concentration was as described above. 


\section{Specific chemical inhibitor study}

To identify the enzymes involved in metabolism, several CYP-specific inhibitors were added to the incubation with rivaroxaban $(120 \mu \mathrm{M})$. The inhibitor concentrations were as follows: 8-mthoxypsoralen $(2.5 \mu \mathrm{M})$ for CYP2A6, montelukast $(5 \mu \mathrm{M})$ for CYP2C8, sulfaphenazole $(10 \mu \mathrm{M})$ for CYP2C9, quinidine $(10 \mu \mathrm{M})$ for CYP2D6, danazol $(3 \mu \mathrm{M})$ for CYP2J2 and ketoconazole $(1 \mu \mathrm{M})$ for the CYP3A subfamily [19-21]. The $120 \mu \mathrm{M}$ rivaroxaban concentration was selected based on the $K_{\mathrm{m}}$ value. The final concentration of organic solvent in the incubation media was no more than $0.5 \%(\mathrm{v} / \mathrm{v})$. Results were from at least three experiments carried out in duplicate.

\section{Molecular docking simulation}

To demonstrate the molecular mechanism between rivaroxaban and CYP2J2, molecular docking simulation was conducted using a CYP2J2 crystal structure homology model. This model was constructed using the Clustal Omega webserver (https://www.ebi.ac.uk/Tools/msa/clustalo/), as previously described [19]. Docking simulation was performed using SYBYL (X-1.1). The 3D structure of rivaroxaban was subjected to energy minimization with the default Tripos force field parameters, and the Gasteiger-Hückel charges were calculated for each compound. The Surflex-Dock mode was used to generate binding conformations of rivaroxaban with CYP2J2, from which the optimal conformations were determined by their empirical functions TotalScore, ChemScore and CScore. In addition, the PyMOL Molecular Graphics System, version 16.1.0.15350 (DeLano Scientific LLC) was used to visualize the docking results.

\section{Results}

Separation and identification of the major metabolite M1

The HPLC conditions to simultaneously detect and separate the major metabolite M1 were methanol:pure water:formic acid $=60: 40: 0.2(\mathrm{v} / \mathrm{v} / \mathrm{v})$, flow rate $0.5 \mathrm{~mL} \cdot \mathrm{min}^{-1}$ and column temperature $30 \mathrm{degC}$. As shown in Figure 2A, when rivaroxaban was incubated with HLM without NADPH, the HPLC peak appeared at 12.839 min. When rivaroxaban was incubated with HLM and NADPH, a new single peak (M1) appeared with a retention time of $10.831 \mathrm{~min}$. The M1 peak was absolutely separate from rivaroxaban and did not interfere with the quantitative analysis, which indicated that the method could be used to simultaneously detect and conduct quantitative analysis of M1 and rivaroxaban.

LC-MS analysis of the major metabolite that was separated and collected from the HPLC was conducted in positive ion mode. Results showed the major metabolite with $\mathrm{m} / \mathrm{z} 452.9$ was the most abundant component with an intensity of $>7 \times 10^{7} \mathrm{cps}$ (Figure 2B). This molecular weight was consistent with results reported in the prior literature; thus, we can confirm that the major metabolite of rivaroxaban was morpholinone 2hydroxylation (M1) (Figure 1) [8].

\section{CYP screening}

Screening experiments with the 17 recombinant enzymes revealed CYP2D6, CYP2J2, CYP3A4 and CYP4F3 to be the four major CYPs for rivaroxaban hydroxylation, as shown by M1 peak area (24.1, 391.6, 25.0 and 14.9), respectively; Figure 3). CYP1A1, CYP3A5 and CYP3A7 also participated in rivaroxaban hydroxylation, but to a lesser extent; however, CYPs 1A2, 1B1, 2A6, 2B6, 2C9, 2C19, 2D6, 2E1, 4F2 and 46A1 were essentially inactive. The catalytic activity of the various CYPs was in the following order: 2J2 > 3A4 $>2 \mathrm{D} 6$ $>4 \mathrm{~F} 3>1 \mathrm{~A} 1>3 \mathrm{~A} 5>3 \mathrm{~A} 7>2 \mathrm{~A} 6>2 \mathrm{E} 1>2 \mathrm{C} 9>2 \mathrm{C} 19$. Notably, CYP2J2 showed the strongest catalytic activity in rivaroxaban hydroxylation of the 17 CYPs, which was approximately 16-, 16- and 26-fold higher than that of CYP2D6, CYP3A4 and CYP4F3, respectively.

\section{Kinetic study}

Given the obvious metabolic activity displayed by CYP2D6, CYP2J2, CYP3A4 and CYP4F3 for rivaroxaban, kinetic studies were conducted to estimate the catalytic capability of HLM (Figure 4A) and these four recombinant CYPs (Figure 4B) in rivaroxaban hydroxylation. All of these reactions obeyed Michaelis-Menten kinetics, as depicted by their respective linear Eadie-Hofstee plots. As shown in Figure 4B, there was a wide 
range in M1 formation rates with the four recombinant CYPs, from 29.42 to 753.9 pmol$^{*} \mathrm{~min}^{-1 *} \mathrm{mg}$ protein ${ }^{-1}$. The $V$ max value of CYP2J2 was $753.9 \mathrm{pmol}^{*} \mathrm{~min}^{-1 *} \mathrm{mg}$ protein ${ }^{-1}$, which was 12.6 -25.6-fold higher than that of the other CYPs (Table 1). Additionally, the apparent $K \mathrm{~m}$ value of CYP2J2 was the lowest among these four recombinant CYPs, which further gave rise to the highest intrinsic clearance value of CYP2J2 $(V \max / K$ $\mathrm{m}=38.92 \mu \mathrm{L} \cdot \mathrm{min}^{-1 *} \mathrm{mg}$ protein $\left.{ }^{-1}\right)$. The intrinsic clearance values indicated that CYP2J2 dominated in the metabolism of rivaroxaban, being approximately 39- to 100-fold higher for CYP2J2 than for the other CYPs. Detailed kinetic parameters are shown in Table 1.

\section{CYP-specific inhibitory study}

To further estimate the contributions of the $\mathrm{P} 450$ isoforms in rivaroxaban hydroxylation, six specific inhibitors were used. As shown in Figure 5, significant inhibition was observed in the groups with CYP3A inhibitor ketoconazole and CYP2J2 inhibitor danazol, reducing enzyme activity to $56.6 \%$ and $58.9 \%$ of control activity, respectively. In addition, CYP2C9-specific inhibitor sulfaphenazole slightly inhibited the formation of M1, remaining about $74.6 \%$ of control. However, inhibition by other inhibitors including quinidine, montelukast and 8-methoxypsoralen was not significant, with the resulting activity remaining at $>90 \%$ that of the control.

\section{Molecular docking simulation}

Molecular docking simulation was used to elucidate the binding conformations of the interaction between rivaroxaban and CYP2J2. The distance between the $\mathrm{O}$ atom of rivaroxaban and the haem iron atom of CYP22 was 3.9 A (Figure 6A). The optimal orientation of rivaroxaban in CYP2J2 and the corresponding 2D ligand interaction diagram are shown in Figure 6. As shown in Figure 6B, the benzene and thiophene rings of rivaroxaban interacted with ALA311 and PHE310, respectively, via $\pi$-alkyl bonds. In addition, alkyl interactions between the methyl group of rivaroxaban and ILE487, VAL380 and ILE376 were apparent. The TotalScore, ChemScore and CScore for rivaroxaban docking to CYP2J2 were 5.35, -27.398 and 4, respectively. Taken together, these results showed a marked binding affinity of rivaroxaban for CYP2J2.

\section{Discussion}

There have been many reports verifying the involvement of CYP3A4 and CYP2J2 in the metabolism of rivaroxaban; however, the respective metabolic contributions of CYP isoforms were unknown. In this study, the contributions of CYP3A4 and CYP2J2 were systematically evaluated and compared. Firstly, CYPdependent M1 formation analysis indicated that CYP2J2 showed a strongest catalytic activity. Moreover, in CYP3A subfamily, except CYP3A4, CYPs 3A5 and 3A7 also participated in the rivaroxaban hydroxylation (M1 area, CYP3A4: CYP3A5: CYP3A7 = 25.0: 8.6: 6.3). Secondly, kinetic studies further verified the highest catalytic efficiency of CYP2J2, approximately 39-fold to that of CYP3A4. Finally, CYP-specific inhibition experiments were used to access the contributions of CYPs. Ketoconazole (CYP3A-specific inhibitor) and danazol (CYP2J2-specific inhibitor) inhibited $43.3 \%$ and $41.1 \%$ rivaroxaban metabolism in HLM. Based on the ratio of M1 area by CYP3A subfamily in CYP screen (M1 area, CYP3A4: CYP3A5: CYP3A7 = 25.0: 8.6: 6.3), the contributions of CYPs $3 \mathrm{~A} 4,3 \mathrm{~A} 5$ and $3 \mathrm{~A} 7$ were $27.3 \%, 9.4 \%$ and $6.9 \%$ respectively. Therefore, our studies identified the predominated role of CYP2J2 in the rivaroxaban hydroxylation with a contribution of $41.1 \%$, which were much higher than the contribution of $27.3 \%$ by CYP3A4.

In the analysis of M1 formation by CYPs, CYP2J2 produced the highest catalytic activity which was nearly 16 times higher than that of CYP3A4 (Figure 3B). Kinetic studies further demonstrated that the intrinsic clearance value of CYP2J2 was far higher than that of CYP3A4, approximately 39-fold (Table 1). Finally, the respective contributions of CYP2J2 and CYP3A4 in HLM were determined by the CYPspecific chemical inhibition study. Even though the inhibitory effect of CYP2J2-specific inhibitor danazol and CYP3A-specific inhibitor ketoconazole were comparable, being $41.1 \%$ and $43.3 \%$ respectively, there were dramatic differences in the content of each isoenzyme in the HLM. CYP3A subfamily is responsible for the metabolism of approximately $30.2 \%$ of clinical drugs, and CYP3A4 as the major isoform of the CYP3A subfamily represents about $14-24 \%$ of the microsomal P450 pool, on average [20-23]. In contrast, CYP2J2 is the least abundant P450 isoform of those involved in clinical drug metabolism [23]. CYP3A was the 
most abundantly-expressed subfamily in the liver, at $\sim 28.8 \%$ of the total hepatic CYPs, whereas CYP2J2 abundance was less than 1\% [23 24]. Moreover, proteomic analysis demonstrated the protein concentration of CYP3A4 to be about 50-fold that of CYP2J2 in HLM [25]. Therefore, we inferred that the results of inhibitory experiments in HLM, CYP2J2 and CYP3A4-specific inhibitors produced comparable inhibition ratio, were most likely caused by the dramatic differences in content for two isoforms in HLM. With such a low content in HLM, CYP2J2 produced a comparable contribution with that by CYP3A, the most abundant subfamily in liver, it suggested the higher catalytic efficiency of CYP2J2 than that of CYP3A4 in rivaroxaban hydroxylation.

In the inhibition study, the total inhibitory activity in HLMs was over $100 \%$ at $130.27 \%$, which may have been due to the poor selectivity of the high-concentration inhibitors. Additionally, the concentration of specific inhibitors had considerable impact on their inhibition selectivity. For example, although ketoconazole is known as a specific inhibitor of the CYP3A subfamily, it has also shown inhibitory activity in the CYP1A1mediated metabolism of 7-ethoxycoumarin, with an $I C$ 50 value of $0.33 \pm 0.03 \mu \mathrm{M}$ [26]. In the present study, the concentration of ketoconazole used for inhibiting CYP3A was set as $1 \mu \mathrm{M}$-which was about $10 \times K_{i}$-to ensure absolute inhibition. At this concentration, ketoconazole may also inhibit CYP1A1, which was also found to be involved in the metabolism of rivaroxaban (Figure 3); this could explain why high concentrations of ketoconazole affected high total inhibition activity. A similar outcome was observed with quinidine, which was used to specifically inhibit the activity of CYP2D6. Quinidine displayed significant inhibition of CYP1A1-mediated 7-ethoxyresorufin O-deethylation, with an $I C$ 50 value of $1.1 \mu \mathrm{M}$ [27]. However, the concentration of quinidine in the present study was set at $10 \mu \mathrm{M}$, which probably also inhibited the CYP1A1mediated metabolism of rivaroxaban and led to a much higher total inhibition activity. Hence, poorly selective inhibitory effects of high-concentration inhibitors resulted in a more than $100 \%$ inhibition ratio in the present study.

In addition to poor selectivity of some inhibitors, the higher expression level of CYP2C9 in the liver may also partly account for its high inhibition. In the CYP-specific inhibition study, the inhibition ratio of CYP2C9 was the third highest after CYP2J2 and CYP3A4, while the M1 peak area produced by CYP2C9 in the CYP screen was less than 10 , and approximately $0.38 \%$ of that produced by CYP2J2. The abundant content of CYP2C9 in the liver played an important role in this difference between the two results. First, CYP2C9 is one of the most highly expressed members of all $\mathrm{P} 450$ isoforms, with a similar or lower protein level to CYP3A4 [22 28]. More importantly, the CYP2C9 protein level was higher than that of CYP3A4 in HLM, and much higher than that of CYP2J2 [25]. Based on this rationale, the inhibition ratio achieved with CYP2C9-specific sulfaphenazole was much higher than its actual efficacy.

The difference in the protein contents of CYP2J2 and CYP3A4 in the liver resulted in their different status in clinical drug-drug interaction research. The drug-drug interactions of rivaroxaban have been extensively assessed in combination with many drugs, including CYP3A4 or P-gp substrates, inhibitors and inducers. However, results have demonstrated that the combination of rivaroxaban with agents that are strong inhibitors of both CYP3A4 and P-gp can increase rivaroxaban plasma concentrations in vivo, prompting caution regarding its co-administration [15]. In contrast, owing to its low expression levels in liver microsomes, CYP2J2 is usually not considered in routine drug-drug interactions, thus, the potential for CYP2J2 regulators to change rivaroxaban clearance has not been widely evaluated to date. However, it is expressed at an extremely high level in the cardiovascular system: an evaluation of the mRNA levels of P450 isoforms in the heart showed CYP2J2 mRNA levels largely exceeded those of other detected isozymes by 3 million to 62 times [29 30]. And, in the aorta and coronary artery, the expression of CYP2J2 ranks second only to CYP2C9 [31]. These distribution characteristics of CYP2J2 are consistent with the function of transforming arachidonic acid into epoxyeicosatrienoic acids, which plays a vital role in cardiovascular homeostasis and regulating vascular tone [32 33]. Importantly, a few rivaroxaban drug-drug interaction studies indicated that it may interact with other cardiovascular drugs that target CYP2J2, such as amiodarone and dronedarone [34]. Therefore, the possibility of the interaction between rivaroxaban and other drugs, especially with drugs to treat cardiovascular diseases, can be more exactly evaluated if the heart is set as the target organ and the content of CYP2J2 is considered. 
In vitro drug-drug interaction (DDI) studies have been one of the major methods for evaluating the efficiency and safety of drugs. For rivaroxaban, the evaluations targeting CYP3A4 and P-gp are the main DDI research direction so far, which was due to the rich content of CYP3A4 in the liver and the key role of P-gp in transporter-mediated DDIs. Our results, showing the dominant role of CYP2J2 in the metabolism of rivaroxaban, fill a gap in the basic metabolism studies of rivaroxaban and also give a new insight into DDI studies involving rivaroxaban.

In summary, multiple CYP isoforms were found to be involved in the hydroxylation of rivaroxaban, with CYP2J2 identified as the predominant isoenzyme involved.

\section{Acknowledgements}

This work was supported by the National Natural Science Foundation of China the National Natural Science Foundation of China (81874324, 81922070, 81973286, 81803627, 81702995 and U1608283), and LiaoNing Revitalization Talents Program (XLYC1907103).

\section{Conflicts of interest}

The authors declare no conflict of interest.

\section{Data availability statement}

The data supporting the findings of the present study are not publicly available; however, on reasonable request, the data are available from the corresponding author.

\section{References}

1. Liakoni E, Rätz Bravo AE, Krähenbühl S. Hepatotoxicity of New Oral Anticoagulants (NOACs). Drug Saf. 2015;38(8):711-20.

2. Yeh CH, Hogg K, Weitz JI. Overview of the new oral anticoagulants: opportunities and challenges. Arterioscler Thromb Vasc Biol.2015;35(5):1056-65.

3. Prins MH, Lensing AW, Bauersachs R, et al. Oral rivaroxaban versus standard therapy for the treatment of symptomatic venous thromboembolism: a pooled analysis of the EINSTEIN-DVT and PE randomized studies. Thrombosis journal. 2013;11(1):21.

4. López-López JA, Sterne JAC, Thom HHZ, et al. Oral anticoagulants for prevention of stroke in atrial fibrillation: systematic review, network meta-analysis, and cost effectiveness analysis. Bmj.2017;359:j5058.

5. Elikowski W, Małek M, Skowroński M, Wróblewski D, Skrzywanek P, Zawilska K. [Hemoptysis during concomitant treatment with rivaroxaban and amiodarone in a patient with a history of pulmonary disease]. Polski merkuriusz lekarski : organ Polskiego Towarzystwa Lekarskiego. 2015;39(232):227-30.

6. Menendez D, Michel J. Hemopericardium with tamponade following rivaroxaban administration and its attenuation by CYP3A4 inhibitors.Proceedings (Baylor University Medical Center). 2016;29(4):414-5.

7. Stöllberger C, Bastovansky A, Finsterer J. Fatal intracerebral bleeding under rivaroxaban. International journal of cardiology.2015;201:110-2.

8. Lang D, Freudenberger C, Weinz C. In vitro metabolism of rivaroxaban, an oral, direct factor Xa inhibitor, in liver microsomes and hepatocytes of rats, dogs, and humans. Drug Metab Dispos. 2009;37(5):1046-55.

9. Weinz C, Schwarz T, Kubitza D, Mueck W, Lang D. Metabolism and excretion of rivaroxaban, an oral, direct factor Xa inhibitor, in rats, dogs, and humans. Drug Metab Dispos. 2009;37(5):1056-64.

10. Dias C, Moore KT, Murphy J, et al. Pharmacokinetics, Pharmacodynamics, and Safety of Single-Dose Rivaroxaban in Chronic Hemodialysis. Am J Nephrol. 2016;43(4):229-36.

11. Gnoth MJ, Buetehorn U, Muenster U, Schwarz T, Sandmann S. In vitro and in vivo P-glycoprotein transport characteristics of rivaroxaban.J Pharmacol Exp Ther. 2011;338(1):372-80. 
12. Mueck W, Kubitza D, Becka M. Co-administration of rivaroxaban with drugs that share its elimination pathways: pharmacokinetic effects in healthy subjects. Br J Clin Pharmacol. 2013;76(3):455-66.

13. EPAR. EU Risk Management Planhttps://wwwemaeuropaeu/en/medicines/human/EPAR/xarelto.2018; [Accessed June 09, 2018].

14. FDA. XareltoXarelto (Rivaroxaban) Product Informationhttps://wwwaccessdatafdagov/drugsatfda_docs/label/2020/202439s031,022406s035lblpdf\#page=60.2020;[Accessed March 01, 2020].

15. Gelosa P, Castiglioni L, Tenconi M, et al. Pharmacokinetic drug interactions of the non-vitamin K antagonist oral anticoagulants (NOACs). Pharmacol Res. 2018;135:60-79.

16. Anand SS, Bosch J, Eikelboom JW, et al. Rivaroxaban with or without aspirin in patients with stable peripheral or carotid artery disease: an international, randomised, double-blind, placebo-controlled trial.Lancet. 2018;391(10117):219-29.

17. Stöllberger C, Zuntner G, Bastovansky A, Finsterer J. Cerebral hemorrhage under rivaroxaban. International journal of cardiology. 2013;167(6):e179-81.

18. Mueck W, Becka M, Kubitza D, Voith B, Zuehlsdorf M. Population model of the pharmacokinetics and pharmacodynamics of rivaroxaban-an oral, direct factor xa inhibitor-in healthy subjects. Int $J$ Clin Pharmacol Ther. 2007;45(6):335-44.

19. Ning J, Liu T, Dong P, et al. Molecular Design Strategy to Construct the Near-Infrared Fluorescent Probe for Selectively Sensing Human Cytochrome P450 2J2. J Am Chem Soc. 2019;141(2):1126-34.

20. Lin YS, Dowling AL, Quigley SD, et al. Co-regulation of CYP3A4 and CYP3A5 and contribution to hepatic and intestinal midazolam metabolism. Mol Pharmacol. 2002;62(1):162-72.

21. Wolbold R, Klein K, Burk O, et al. Sex is a major determinant of CYP3A4 expression in human liver. Hepatology. 2003;38(4):978-88.

22. Ohtsuki S, Schaefer O, Kawakami H, et al. Simultaneous absolute protein quantification of transporters, cytochromes P450, and UDP-glucuronosyltransferases as a novel approach for the characterization of individual human liver: comparison with mRNA levels and activities. Drug Metab Dispos. 2012;40(1):83-92.

23. Zanger UM, Schwab M. Cytochrome P450 enzymes in drug metabolism: regulation of gene expression, enzyme activities, and impact of genetic variation. Pharmacol Ther. 2013;138(1):103-41.

24. Michaels S, Wang MZ. The revised human liver cytochrome P450 "Pie": absolute protein quantification of CYP4F and CYP3A enzymes using targeted quantitative proteomics. Drug Metab Dispos.2014;42(8):124151.

25. Wang X, He B, Shi J, Li Q, Zhu HJ. Comparative Proteomics Analysis of Human Liver Microsomes and S9 Fractions. Drug Metab Dispos.2020;48(1):31-40.

26. Sai Y, Dai R, Yang TJ, et al. Assessment of specificity of eight chemical inhibitors using cDNA-expressed cytochromes P450.Xenobiotica; the fate of foreign compounds in biological systems.2000;30(4):327-43.

27. Ching MS, Blake CL, Malek NA, Angus PW, Ghabrial H. Differential inhibition of human CYP1A1 and CYP1A2 by quinidine and quinine.Xenobiotica; the fate of foreign compounds in biological systems.2001;31(11):757-67.

28. Naraharisetti SB, Lin YS, Rieder MJ, et al. Human liver expression of CYP2C8: gender, age, and genotype effects. Drug Metab Dispos.2010;38(6):889-93.

29. Wu S, Moomaw CR, Tomer KB, Falck JR, Zeldin DC. Molecular cloning and expression of CYP2J2, a human cytochrome P450 arachidonic acid epoxygenase highly expressed in heart. J Biol Chem.1996;271(7):3460-8. 
30. Michaud V, Frappier M, Dumas MC, Turgeon J. Metabolic activity and mRNA levels of human cardiac CYP450s involved in drug metabolism.PLoS One. 2010;5(12):e15666.

31. Delozier TC, Kissling GE, Coulter SJ, et al. Detection of human CYP2C8, CYP2C9, and CYP2J2 in cardiovascular tissues. Drug Metab Dispos. 2007;35(4):682-8.

32. Node K, Huo Y, Ruan X, et al. Anti-inflammatory properties of cytochrome P450 epoxygenase-derived eicosanoids. Science.1999;285(5431):1276-9.

33. Xu X, Zhang XA, Wang DW. The roles of CYP450 epoxygenases and metabolites, epoxyeicosatrienoic acids, in cardiovascular and malignant diseases. Adv Drug Deliv Rev. 2011;63(8):597-609.

34. Cheong EJ, Goh JJ, Hong Y, et al. Application of Static Modeling -in the Prediction of In Vivo DrugDrug Interactions between Rivaroxaban and Antiarrhythmic Agents Based on In Vitro Inhibition Studies. Drug Metab Dispos. 2017;45(3):260-8.

Table 1 . Kinetic parameters of rivaroxaban by HLM and selected CYPs.

\begin{tabular}{lllll}
\hline & & & \multicolumn{1}{c}{$\begin{array}{l}C L_{\text {int fold }} \\
\text { (CYP2J2 } /\end{array}$} \\
Enzyme source & $K_{\mathrm{m}}$ & $V_{\max }$ & $C L_{\text {int }}$ & CYPs) \\
\hline HLM & $\mathbf{2 2 . 8 1} \pm \mathbf{2 . 3 7 0}$ & $\mathbf{9 1 . 2 8} \pm \mathbf{3 . 2 3 3}$ & 4.002 & - \\
CYP2J2 & $\mathbf{1 9 . 3 7} \pm \mathbf{1 . 9 3 2}$ & $\mathbf{7 5 3 . 9} \pm \mathbf{1 9 . 9 7}$ & $\mathbf{3 8 . 9 2}$ & - \\
CYP3A4 & $46.98 \pm 4.800$ & $\mathbf{4 7 . 2 4} \pm 1.843$ & $\mathbf{1 . 0 0 6}$ & $\mathbf{3 8 . 6 8}$ \\
CYP2D6 & $\mathbf{9 8 . 7 8} \pm \mathbf{8 . 8 3 9}$ & $\mathbf{5 9 . 9 4} \pm \mathbf{2 . 5 9 7}$ & $\mathbf{0 . 6 0 7}$ & $\mathbf{9 9 . 1 1}$ \\
CYP4F3 & $\mathbf{7 5 . 1 9} \pm \mathbf{5 . 3 1 2}$ & $\mathbf{2 9 . 4 2} \pm \mathbf{0 . 8 9 8}$ & $\mathbf{0 . 3 9 1}$ & \\
\hline
\end{tabular}

$K_{\mathrm{m}}$ was recorded as $\mu \mathrm{M} ; V_{\max }$ was recorded as $\mathrm{pmol} \cdot \mathrm{min}^{-1} \cdot \mathrm{mg} \operatorname{protein}^{-1} ; C L_{\mathrm{int}}\left(V \max / K_{\mathrm{m}}\right)$ was recorded as $\mu \mathrm{L} \cdot \mathrm{min}^{-1} \cdot \mathrm{mg}$ protein ${ }^{-1}$. The data is reported as mean \pm S.D. of at least three incubations performed in duplicate.

Figure legand

Figure. 1. CYP-dependent oxidation of rivaroxaban to hydroxylated rivaroxaban.

Figure. 2. (A) HPLC profile of rivaroxaban and metabolite M1 in the presence or absence of NADPH. (B) Total ion chromatograms of metabolite M1.

Figure. 3. M1 formation catalyzed by various recombinant human CYPs. Rivaroxaban $(120 \mu \mathrm{M})$ was incubated with human CYPs $\left(0.4 \mathrm{mg} \cdot \mathrm{mL}^{-1}\right)$ at $37 \mathrm{deg}$ for 60 minutes. Results are shown as the mean +- S.D. of at least three determinations performed in duplicate. N.D.: Not detectable.

Figure. 4 . Kinetic plots of rivaroxaban metabolism after incubation with (A) HLM; (B) CYP2D6, CYP2J2, CYP3A4 and CYP4F3. Inset: Eadie-Hofstee transformation.

Figure. 5 . The effect of P450-specific inhibitors on rivaroxaban hydroxylation in HLM. The data are shown as mean +- S.D. from three independent experiments performed in duplicate. ${ }^{*} P<0.05$ and $^{* *} P<$ 0.01 vs the control group; ns: not significant $(P>0.05$ vs the control group).

Figure. 6 . (A) Conformation of rivaroxaban with the iron in porphyrin ring of CYP2J2 and (B) corresponding 2D molecular interaction diagram.

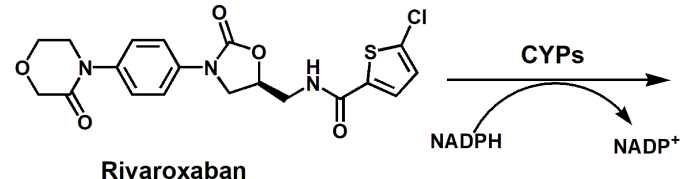

Rivaroxaban

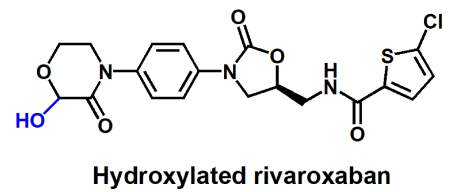

Hydroxylated rivaroxaban 
A

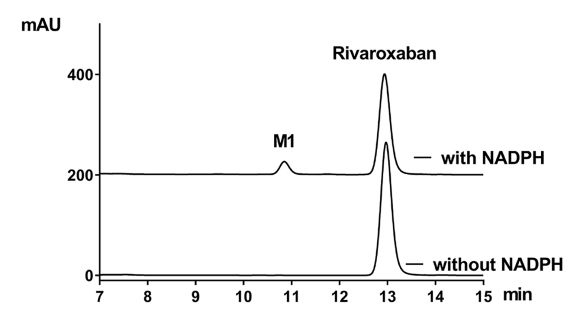

B

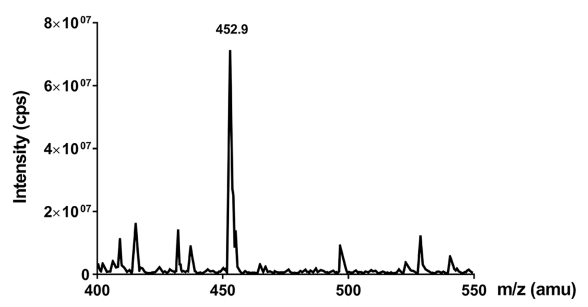

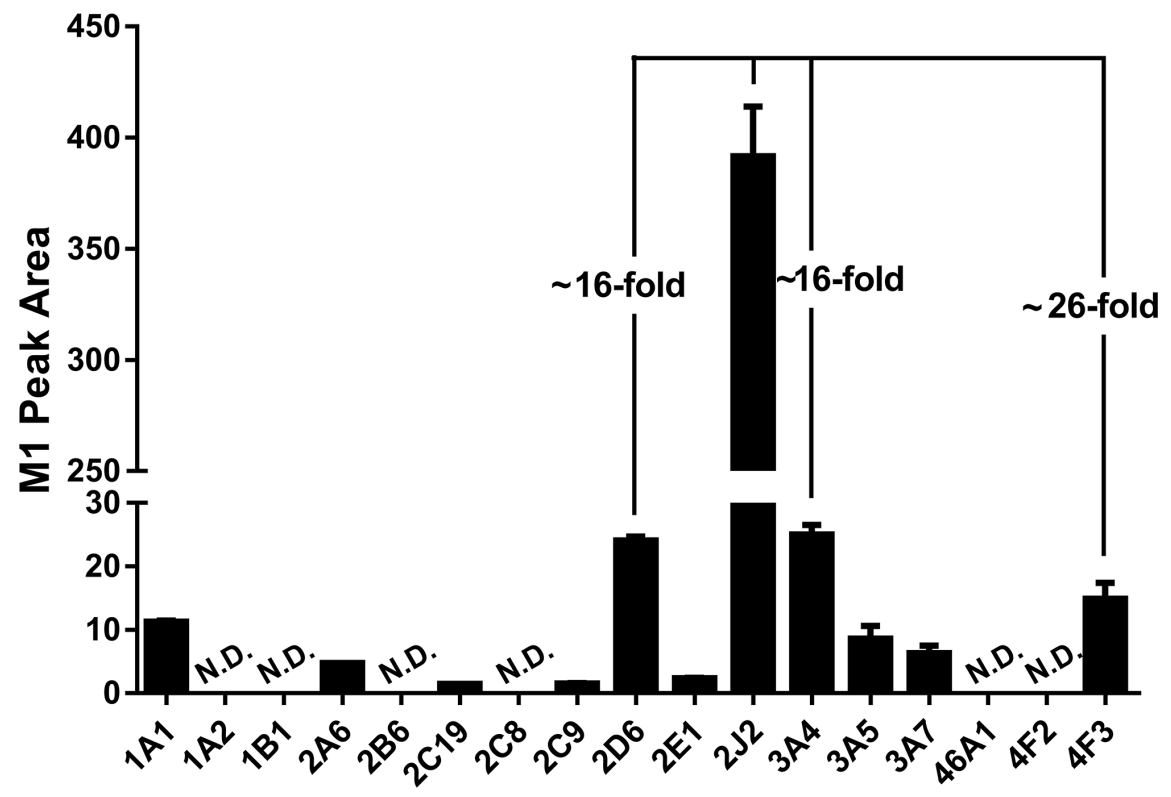

A

B
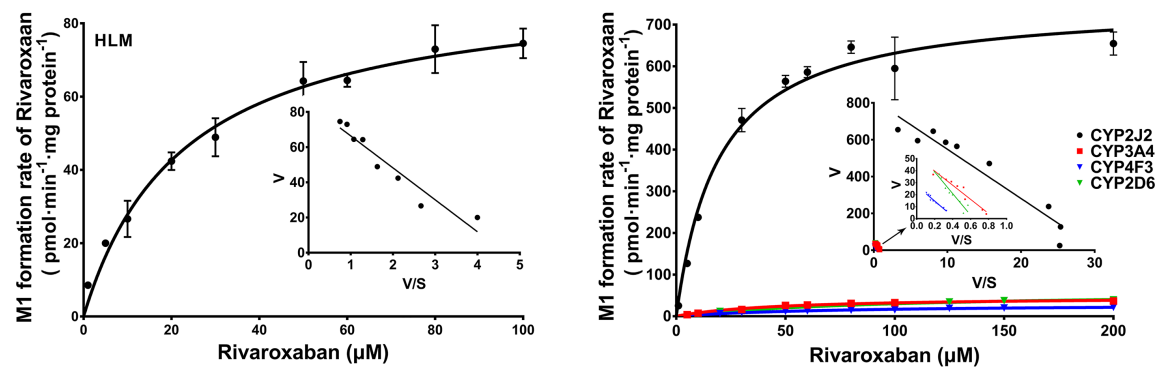


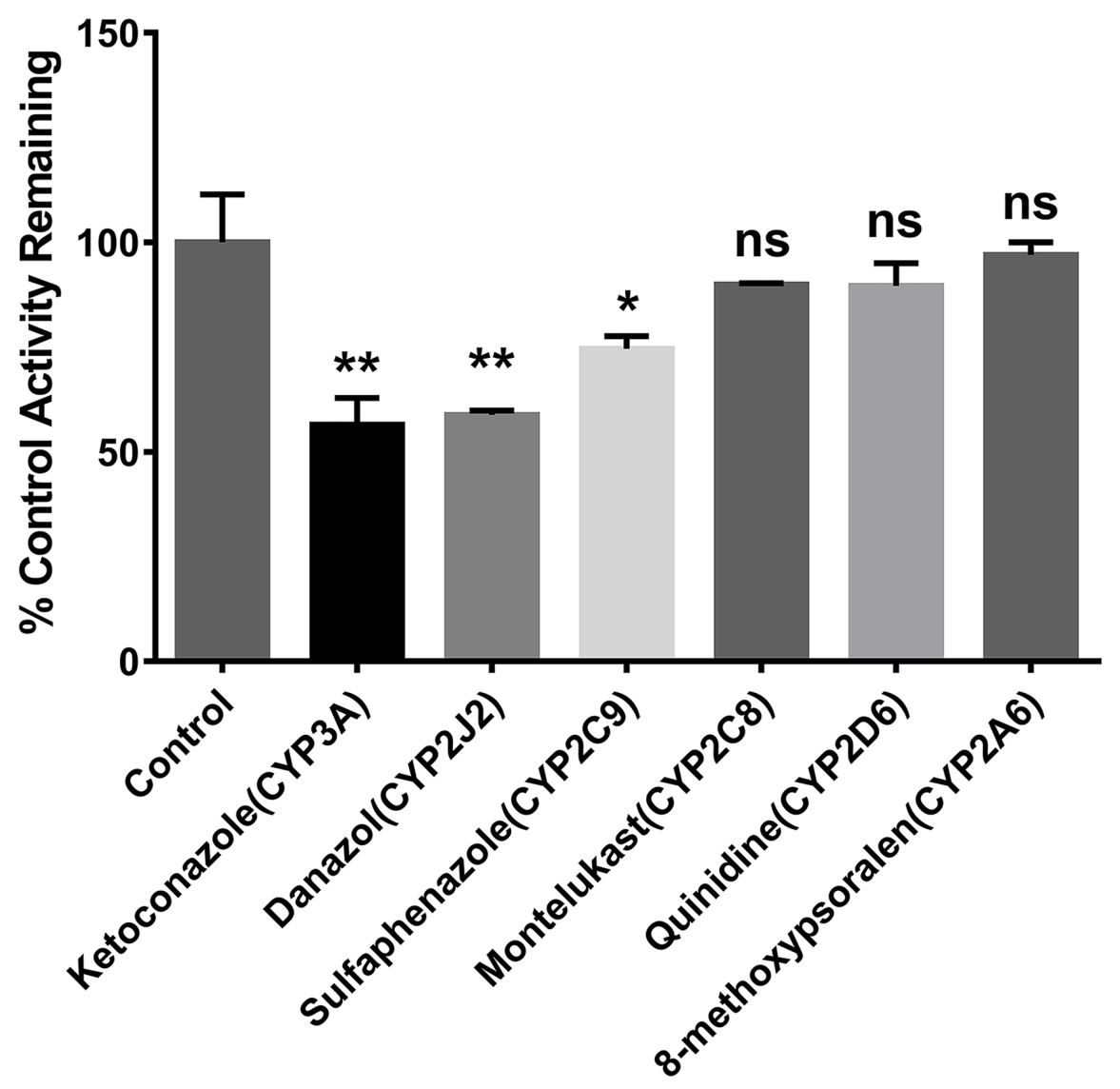

A

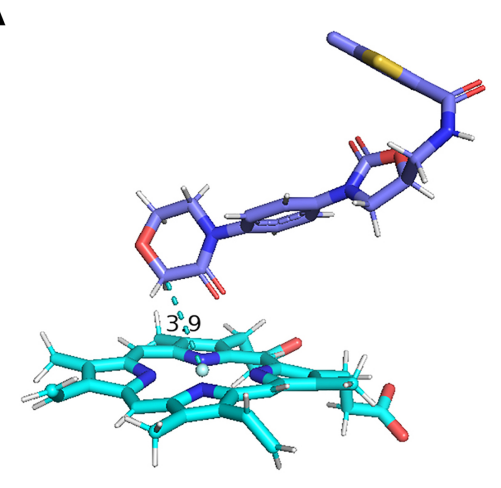

B

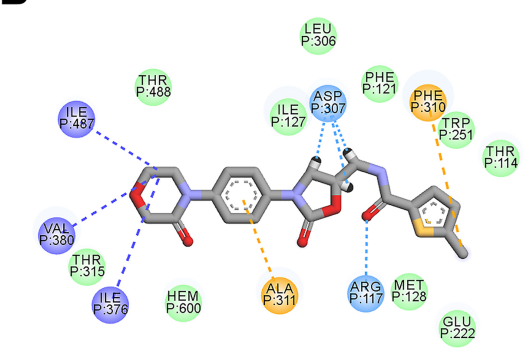

Interactions

$\square$ van der Waals
Carbon Hydrogen Bond
Alkyl 